\title{
EXPERIMENTAL AND NUMERICAL ANALYSIS OF THE INFLUENCE OF EMBEDDING THICKNESS IN COMPRESSIVE MECHANICAL TESTING OF VERTEBRAL AUGMENTATION AND SPINAL INTERBODY FUSION
}

\author{
Márta Kurutz ${ }^{1}$, Péter Nédli ${ }^{1}$, Péter Varga ${ }^{2}$, Tibor Csákány ${ }^{3}$, Gábor Jakab ${ }^{3}$ \\ ${ }^{1}$ Budapest University of Technology and Economics \\ 2Julius Wolff Institute and Berlin-Brandenburg School for Regenerative Therapies, Charitè - \\ Universitätsmedizin \\ ${ }^{3}$ National Center for Spinal Disorders, Budapest \\ kurutrm@eik.bme.bu
}

\begin{abstract}
For in vitro mechanical testing of biomechanical objects the specimens must generally be embedded into a special material to obtain a stable position in the testing machine. However, the experimental boundary conditions may influence the results of the measurements. In this study the effect of embedding thickness on the mechanical properties of vertebrae treated by vertebroplasty and kyphoplasty and lumbar motion segments treated by interbody fusion with PEEK and PMMA cement spacers is analyzed by using compressive mechanical tests and QCT based specimen-specific finite element simulation.
\end{abstract}

Keywords: mechanical compressive test, embedding thickness, vertebroplasty, kyphoplasty, interbody fusion, specimen-specific finite-element method

\section{Introduction}

In vitro mechanical tests combined with computational methods are widely used for comprehensive analysis of spinal operation techniques. Mechanical testing of spinal specimens can be carefully well-prepared, however, to measure the mechanical characteristics and effective tissue moduli of the objects may have some uncertainties and additional errors due to the experimental boundary conditions, namely, due to the differences between model and experiment, in particular how the experimental boundary conditions are modelled. ${ }^{1}$ Among others, material and embedding techniques highly influence the measured values, mainly for cellular structures. To determine the dependence of effective tissue properties on the applied boundary conditions and quantifying errors was investigated in ${ }^{1,2}$ by using finite element method. According to our observations during comprehensive mechanical tests of augmented vertebrae and interbody fixation, changing the thickness of the embedding of specimens, the mechanical properties like failure loads, displacements and elastic stiffnesses can significantly change.

In this study the effect of embedding thickness on the mechanical properties of vertebrae treated by vertebroplasty and kyphoplasty and lumbar motion segments treated by interbody fusion with PEEK and PMMA cement spacers is analyzed by using compressive mechanical tests and QCT based specimen-specific finite element simulation. 


\section{Methods}

The specimens were prepared in the National Center for Spinal Disorders in Budapest, and the compression tests were executed in the laboratory of the Biomechanical Research Centre of the Budapest University of Technology and Economics.

For the vertebral augmentation specimens 37 lumbar vertebrae were extracted from 11 human female cadaveric lumbar spines (spine/level/gender/age): A/L1-L4/F/60; B/L1-L3/F/60; C/L1-L3/-/-; D/T12-L5F/51; E/L1-L4/F/57; F/L1-L4/F/95; G/L1-L4/-/-; H/L1-L4/F/80; I/L1-L4/F/70; J/L1-L5F/60; K/L1-L5/F/88. The 37 vertebrae were divided into 2 groups: 16 vertebrae for vertebroplasty (VP) and 21 vertebrae for kyphoplasty (KP). In the VP group a total volume of $6 \mathrm{ml}$ of PMMA cement was injected, in 3-3 $\mathrm{ml}$ bipedicular way. Similarly, in the KP group, 3-3 ml PMMA cement was injected into the place of inflated and removed two balloons, yielding a total value of $6 \mathrm{ml}$, as well. Thus, the same amount of cement was injected into all VP and KP augmented vertebrae. The VP and KP groups were further divided into two groups: 8 vertebra from VP group for thick embedding (VP1) group and 8 for thin embedding (VP2) group; 10 vertebra from KP group for thick embedding (KP1) group and 11 for thin embedding (KP2) group. The specimens of thick and thin embedding groups were embedded parallel to about 8,5 and 3,5 mm thick polymethylmethacrylate PMMA plastic discs, respectively, around the inferior and superior endplates of vertebrae. In Table $1 e 1 a$ and $1 b$ the embedding thickness data of thick and thin embedded groups are shown. The pre-operated detailed data of vertebrae can be seen in ${ }^{3}$ published in the present proceedings book as well.

Thick embedded groups (VP1 and KP1)

\begin{tabular}{|c|c|c|c|c|c|c|c|}
\hline Specimen & Sample & $\begin{array}{c}\text { Superior } \\
\text { embedding } \\
\text { thickness } \\
(\mathrm{mm})\end{array}$ & $\begin{array}{c}\text { Inferior } \\
\text { embedding } \\
\text { thickness } \\
(\mathrm{mm})\end{array}$ & Specimen & Sample & $\begin{array}{c}\text { Superior } \\
\text { embedding } \\
\text { thickness } \\
(\mathrm{mm})\end{array}$ & $\begin{array}{c}\text { Inferior } \\
\text { embedding } \\
\text { thickness } \\
(\mathrm{mm})\end{array}$ \\
\hline VP1 group & & & & KP1 group & & & \\
\hline VP1/1 & A/L1 & 6.61 & 4.70 & KP1/1 & $\bar{A} / \mathrm{L} 2$ & 7.52 & 6.11 \\
\hline VP1/2 & A/L3 & 4.70 & 7.52 & $\mathrm{KP} 1 / 2$ & A/L4 & 5.17 & 5.64 \\
\hline VP1/3 & B/L1 & 7.99 & 10.35 & $\mathrm{KP} 1 / 3$ & B/L2 & 8.46 & 8.93 \\
\hline VP1/4 & B/L3 & 10.82 & 7.05 & KP1/4 & C/L1 & 7.52 & 5.66 \\
\hline VP1/5 & $\mathrm{C} / \mathrm{L} 2$ & 9.87 & 6.58 & $\mathrm{KP} 1 / 5$ & $\mathrm{C} / \mathrm{L} 3$ & 7.53 & 7.05 \\
\hline VP1/6 & $\mathrm{D} / \mathrm{L} 1$ & 6.11 & 4.23 & $\mathrm{KP} 1 / 6$ & D/T12 & 12.05 & 10.82 \\
\hline VP1/7 & D/L3 & 9.45 & 9.40 & KP1/7 & D/L2 & 10.44 & 10.34 \\
\hline VP1/8 & D/L5 & 11.29 & 10.82 & $\mathrm{KP} 1 / 8$ & D/L4 & 10.35 & 12.70 \\
\hline Mean/SD & & $8.89 / 2.19$ & $7.84 / 2.42$ & Mean/SD & & $8.63 / 2.19$ & $8.41 / 2.69$ \\
\hline
\end{tabular}

Table 1a. Embedding thickness values of pre-operated specimens for the thick embedded groups 
Thin embedded groups (VP2 and KP2)

\begin{tabular}{|c|c|c|c|c|c|c|c|}
\hline Specimen & Sample & $\begin{array}{c}\text { Superior } \\
\text { embedding } \\
\text { thickness } \\
(\mathrm{mm})\end{array}$ & $\begin{array}{c}\text { Inferior } \\
\text { embedding } \\
\text { thickness } \\
(\mathrm{mm})\end{array}$ & Specimen & Sample & $\begin{array}{c}\text { Superior } \\
\text { embedding } \\
\text { thickness } \\
(\mathrm{mm})\end{array}$ & $\begin{array}{c}\text { Inferior } \\
\text { embedding } \\
\text { thickness } \\
(\mathrm{mm})\end{array}$ \\
\hline VP2 group & & & & KP2 group & & & \\
\hline VP2/1 & E/L1 & 3.33 & 4.00 & KP2/1 & E/L2 & 4.00 & 4.00 \\
\hline $\mathrm{VP} 2 / 2$ & E/L3 & 3.00 & 3.68 & KP2/2 & $\mathrm{E} / \mathrm{L} 4$ & 5.00 & 4.00 \\
\hline VP2/3 & G/L2 & 3.33 & 3.72 & $\mathrm{KP} 2 / 3$ & G/L3 & 3.00 & 3.68 \\
\hline $\mathrm{VP} 2 / 4$ & I/L1 & 3.29 & 4.26 & KP2/4 & I/L2 & 3.29 & 2.82 \\
\hline VP2/5 & I/L3 & 2.40 & 3.42 & $\mathrm{KP} 2 / 5$ & I/L4 & 2.82 & 2.82 \\
\hline VP2/6 & $\mathrm{H} / \mathrm{L} 2$ & 2.82 & 2.82 & KP2/6 & K/L1 & 4.70 & 3.76 \\
\hline VP2/7 & $\mathrm{J} / \mathrm{L} 3$ & 4.70 & 2.82 & KP2/7 & H/L1 & 3.29 & 3.32 \\
\hline $\mathrm{VP} 2 / 8$ & J/L5 & 3.76 & 3.76 & $\mathrm{KP} 2 / 8$ & J/L1 & 4.23 & 3.29 \\
\hline VP2/9 & F/L2 & 3.29 & 3.32 & KP2/9 & $\mathrm{J} / \mathrm{L} 4$ & 3.29 & 2.82 \\
\hline \multirow[t]{2}{*}{ VP2/10 } & F/L4 & 4.23 & 2.82 & KP2/10 & F/L2 & 4.26 & 3.12 \\
\hline & & & & KP2/11 & F/L4 & 4.70 & 3.76 \\
\hline Mean/DS & & $3.40 / 0.64$ & $3.49 / 0.50$ & Mean/SD & & $3.87 / 0.76$ & $3.40 / 0.47$ \\
\hline
\end{tabular}

Table $1 \mathrm{~b}$. Embedding thickness values of pre-operated specimens for the thin embedded groups

For the lumbar interbody fusion specimens 16 cadaveric lumbar motion segments were extracted from 8 human lumbar spines (spine/level/gender/age/BMI/): A/L1-4/M/63/24.5/; B/L14/M/52/27.1/; C/L1-4/F/79/24.2/; D/L1-4/M/64/24.5/; E/L1-4/F/88/28.6/; F/L14/F/88/24.2/; G/L1-4/F/75/24.7/; H/L1-4/M/56/24.7/ that were scanned with dual energy $\mathrm{X}$-ray absorptiometry (DEXA) to obtain bone mineral density (BMD), T-score and Z-score. The 16 motion segments were divided into 2 groups: 8 specimens with PEEK spacers and 8 with PMMA cement cages. In the PMMA group, all possible free places of the intervertebral space were filled in by the injected cement. No further filling material or bone debris was used for the present experiments. The specimens of both groups were embedded parallel by equally about 10 mm thick PMMA plastic discs around the inferior and superior endplates of the inferior and superior vertebrae, respectively. The pre-operated detailed data of the segments can be seen in ${ }^{4}$ published in the present proceedings book as well.

Both the pre- and post-operated specimens were scanned individually with a high-resolution quantitative computed tomography (QCT) system (Hitachi Presto, Hitachi Medical Corporation, Tokyo, Japan) to provide 3D density maps of the vertebral bodies. The samples were scanned in native state submerged in a water filled box. The PMMA embedding of specimens were transparent in CT to distinguish correctly the bone and embedding, so the bordering planes of embeddings were marked by glass pearls. Vertebral heights, central cross sectional areas, CT grey values were measured from QCT images. The CT scan was performed for each specimen after the mechanical test as well. The specimens were stored at $-200 \mathrm{C}$ and were thawed at room temperature 4-6 hours before testing.

Compressive mechanical testing of the specimens was performed by using a servohydraulic testing machine (Instron 8870, Norwood, USA). Axial compressive displacement load was applied at a rate of $5 \mathrm{~mm} / \mathrm{min}$ speed, to the limit of $20 \%$ decrease of the compressive force or 
$20 \%$ of compressive strain of the specimen. Axial compressive force and displacement were measured and the relating force-displacement curve was plotted. Failure load, failure displacement and structural stiffness were extracted from the load-displacement curves. Failure load was the maximum load before the gradient of the curve changed from positive to negative, whereas stiffness was the slope of the linear portion of the load-displacement curve before failure occurred. Dividing the failure displacements by the original heights, failure strains were also calculated for vertebra specimens.

At the same time, CT-based case-specific finite element models were constructed detailed in ${ }^{5}$ published in the present proceedings book. The post-operative CT images were segmented by means of a semi-automated approach using the software tool ZibAmira (Zuse Institute Berlin, Germany). For the fixed motion segments the following volumes were distinguished: cortical and trabecular bone of both vertebral bodies, the articular cartilage layers at the joints of the posterior elements, the remnants of the disc annulus as well as the PEEK or the PMMA cement spacer, respectively. For the augmented vertebrae the following compartments were separated: cortical and trabecular bone volumes of vertebrae included the PMMA cement augmentation volume of vertebroplasty or kyphoplasty. The embedding layers were added as rectangular regions indicated by the glass bead positions.

The finite element meshes of each motion segment and augmented vertebra were then generated based on these images; all domains were meshed with linear tetrahedral elements using the CGAL library (www.cgal.org). Maximal cell size was set to $1.4 \mathrm{~mm}$ and the size of the tetrahedrons was adjusted to the local dimensions of the geometrical features. The finite element simulations were performed in Abaqus v6.10 (SIMULIA, Dassault Systemes, Velizy-Villacoublay, France) and ANSYS v14.0 (ANSYS Inc., Southpointe, USA).

Material properties of the soft tissues, spacer, cement and embedding were assumed to be isotropic, homogeneous and linear elastic. For the PMMA cement and plastic materials the elastic modulus was measured experimentally on small cylindrical samples. The properties of PEEK, articular cartilage and annulus were taken from the literature. Bone was assumed to be a transversely isotropic, inhomogeneous and linear elasto-plastic material. For both the trabecular and cortical bone compartments the properties of the elements were scaled based on the CT images by converting the Hounsfield (HU) values into bone mineral density (BMD) units, detailed in. ${ }^{5}$ The elements were categorized into 255 sets based on their mean densities. For each set, the elastic moduli and the yield strength were computed using experimentally established vertebra-specific relationships ${ }^{8}$ based on. ${ }^{6-7}$ The material properties used in the finite element models are seen in Table 1.

\begin{tabular}{|l|c|c|c|c|}
\hline \multicolumn{1}{|c|}{ Material } & Type & Anisotropy & $\begin{array}{c}\text { Young's mod } \\
{[\mathrm{MPa}]}\end{array}$ & $\begin{array}{c}\text { Poisson's } \\
\text { ratio }\end{array}$ \\
\hline bone & linear elasto-plastic & transversely isotropic & BMD-based & 0.381 and 0.104 \\
\hline disc annulus & linear elastic & isotropic & 10 & 0.45 \\
\hline articular cartilage & linear elastic & isotropic & 5 & 0.45 \\
\hline bone cement & linear elastic & isotropic & 10000 & 0.3 \\
\hline embedding plastic & linear elastic & isotropic & 700 & 0.3 \\
\hline PEEK spacer & linear elastic & isotropic & 4000 & 0.4 \\
\hline
\end{tabular}

Table 2. Material properties used in the finite element model 
As for the boundary conditions of the model, the embedding layers, the augmentation cement, the PMMA bone cement and PEEK spacer were assumed to be rigidly bound to the bone surfaces. At present, as a first simplified solution the cartilage layer was assumed to be very soft. The lower plane of the inferior embedding layer was fully constrained. The displacement load was applied on the upper surface of the superior embedding along the direction of the plane normal.

In the finite element analysis of the effect of embedding thickness on the mechanical behaviour of the interbody fusion, we applied a slicing method. The original $10 \mathrm{~mm}$ thickness of the embedding was gradually made thinner by removing $2.5 \mathrm{~mm}$ thick slices from the embedding just to the total removal of it. These steps were made first parallel in the superior and the inferior embeddings, then separately. All these sliced versions were checked under inverse boundary conditions and loading, namely, first supported down and loaded from the top and later inversely.

In this paper, the effect of embedding thickness will be presented only for the mechanical tests of augmented vertebrae and for the finite element analysis of the plastic damage process of a single case of interbody fusion using PMMA cement spacer. The comparative analysis using both mechanical test and case-specific finite element simulation for both vertebral augmentation and interbody fusion is under progress.

\section{Results}

\subsection{The influence of the embedding thickness on the mechanical characteristics of vertebroplasty and kyphoplasty during compressive mechanical test}

The results of the compressive mechanical tests, the values of the failure load and displacement and the elastic stiffness are detailed $i^{3}$ published in the present proceedings book. Here we cite the results relating to the embedding only. In Figure 1 the mean failure load, stiffness and failure height loss of VP and KP groups are shown with thick and thin embeddings.

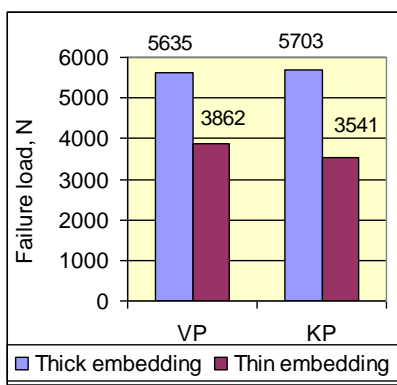

a)

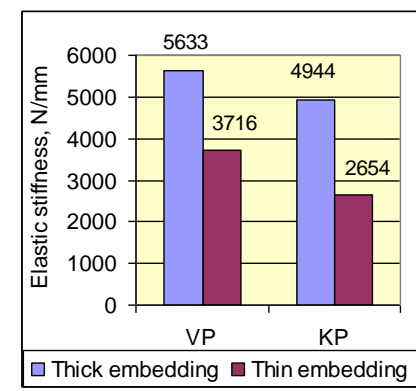

b)

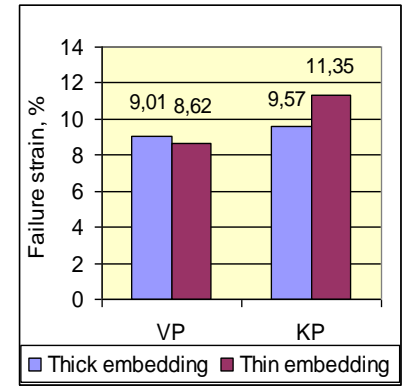

c)

Figure 1. Mean failure load (a), stiffness (b) and failure height loss (c) of VP and KP groups with thick and thin embeddings

Compressive failure load was practically equal for VP and KP groups in thick $(\mathrm{P}=0.40)$ embedded group and was about $8 \%$ smaller in thin embedded groups $(\mathrm{P}=0.31)$ for KP vertebrae, 
compared to the VP ones (Figure 1a). Compressive stiffness for KP treatment was $12 \%$ smaller in thick $(\mathrm{P}=0.18)$ and $29 \%$ smaller in thin $(\mathrm{P}=0.02)$ embedded groups, compared to the VP ones (Figure 1b). Compressive strain was $6 \%$ larger in the thick $(\mathrm{P}=0.35)$ and $32 \%$ larger in thin $(\mathrm{P}=0.018)$ embedded groups for KP than for VP augmentation (Figure 1c). The differences between the mechanical properties of VP and KP augmentation were significantly higher in the thin embedded group $(\mathrm{P}=0.04)$.

The embedding thickness significantly affected most of the mechanical results inside the augmentation type as well. The failure load of the thin embedded groups was $32 \%$ smaller for $\mathrm{VP}(\mathrm{P}=0.018)$ and $38 \%$ smaller for $\mathrm{KP}(\mathrm{P}=0.0016)$ vertebrae compared to the thick embedded groups (Figure 1a). Elastic stiffness of the thin embedded groups was 34\% smaller for VP $(\mathrm{P}=0.031)$ and $46 \%$ smaller for KP $(\mathrm{P}=0.0006)$ vertebrae compared to the thick embedded groups (Figure 1b). However, failure strain of the thin embedded groups was only $4 \%$ smaller for VP $(\mathrm{P}=0.45)$ but $19 \%$ higher for $\mathrm{KP}$ vertebrae $(\mathrm{P}=0.021)$, compared to the thick embedded groups (Figure 1c).

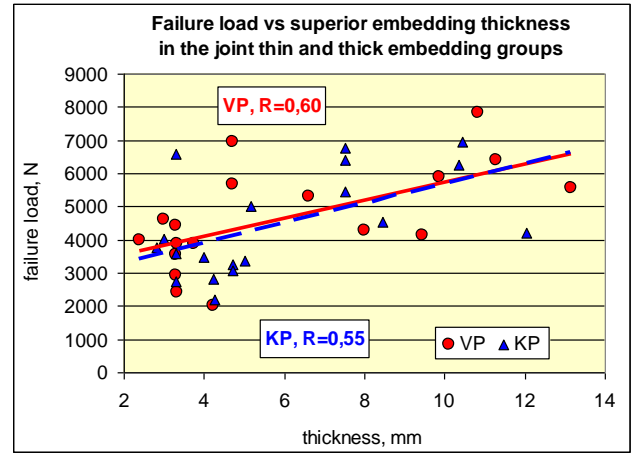

a)

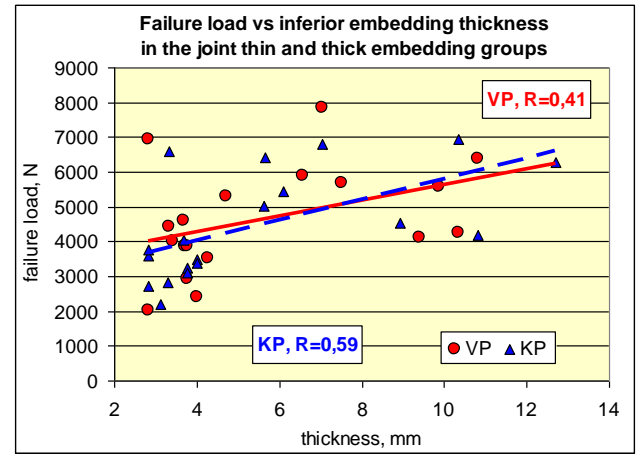

b)

Figure 2. Failure load vs a) superior and b) inferior embedding thickness in VP and KP groups

\begin{tabular}{|c|c|c|c|c|c|c|}
\hline $\begin{array}{c}\text { Failure load vs } \\
\text { emb. thickness }\end{array}$ & VP1 & KP1 & VP2 & KP2 & VP & KP \\
\hline superior & 0.31 & -0.07 & 0.28 & -0.41 & 0.60 & 0.55 \\
\hline inferior & -0.26 & -0.02 & -0.35 & 0.05 & 0.41 & 0.59 \\
\hline mean & 0.07 & -0.04 & 0.03 & -0.26 & 0.54 & 0.58 \\
\hline
\end{tabular}

Table 3. Correlation between failure load and superior, inferior and mean embedding thickness

Correlation between the failure load and the embedding thicknesses of thick and thin embedded VP and KP vertebrae can be seen in Figure 2 and Table 3. Evidently, while the failure load of both VP and KP vertebrae showed practically no correlation with the embedding thickness both in thick and thin embedded groups of small thickness differences, in the joint thick and thin embedded groups of radical thickness differences a good positive correlation was observed, 
equally for the superior $(R=0.60, R=0.55)$, inferior $(R=0.41, R=0.59)$ and mean $(R=0.54, R=0.58)$ embedding thicknesses, for VP and KP vertebrae, respectively.

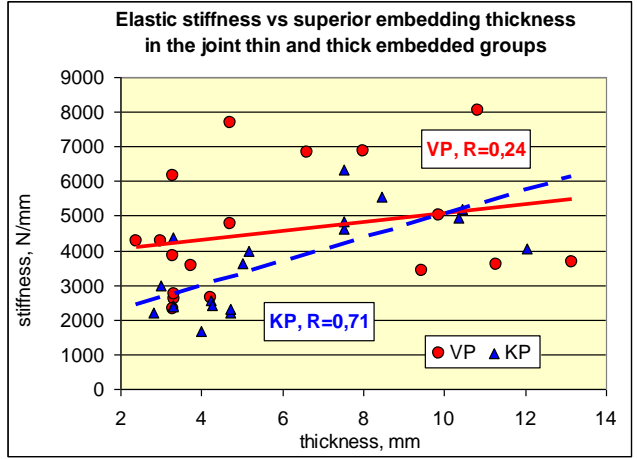

a)

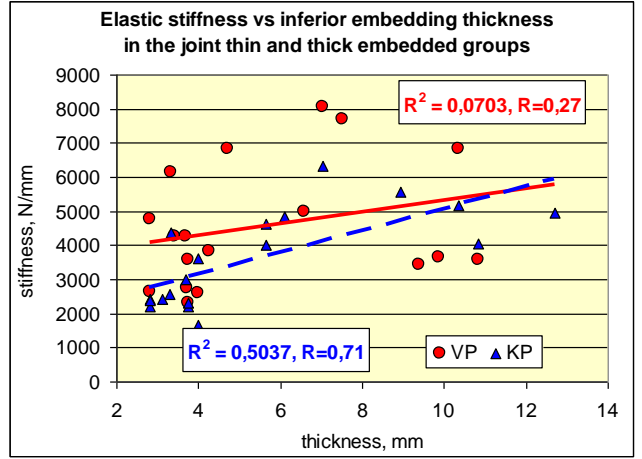

b)

Figure 3. Elastic stiffness vs a) superior and b) inferior embedding thickness in VP and KP groups

\begin{tabular}{|c|c|c|c|c|c|c|}
\hline $\begin{array}{c}\text { Stiffness vs } \\
\text { emb. thickness }\end{array}$ & VP1 & KP1 & VP2 & KP2 & VP & KP \\
\hline superior & -0.62 & -0.05 & -0.04 & -0.06 & 0.24 & 0.71 \\
\hline inferior & -0.55 & -0.03 & -0.28 & 0.08 & 0.27 & 0.71 \\
\hline mean & -0.69 & 0.00 & -0.30 & 0.01 & 0.26 & 0.72 \\
\hline
\end{tabular}

Table 4. Correlation between elastic stiffness and superior, inferior and mean embedding thickness

Correlation between the elastic stiffness and the embedding thicknesses of thick and thin embedded VP and KP vertebrae can be seen in Figure 3 and Table 4. While the stiffness of VP vertebrae showed a considerable negative correlation with the embedding thickness both in thick and thin embedded groups, in the joint thick and thin embedded groups of radical thickness differences a modest positive correlation was observed for the superior $(R=0.24)$, inferior $(\mathrm{R}=0.27)$ and mean embeddings $(\mathrm{R}=0,26)$. However, while the stiffness of $\mathrm{KP}$ vertebrae showed no correlation in separated thin and thick embedded groups, for the joint groups a strong positive correlation was observed equally for the superior $(\mathrm{R}=0.71$, inferior $(\mathrm{R}=0.71)$ and nean $(\mathrm{R}=0.72)$ embedding thicknesses

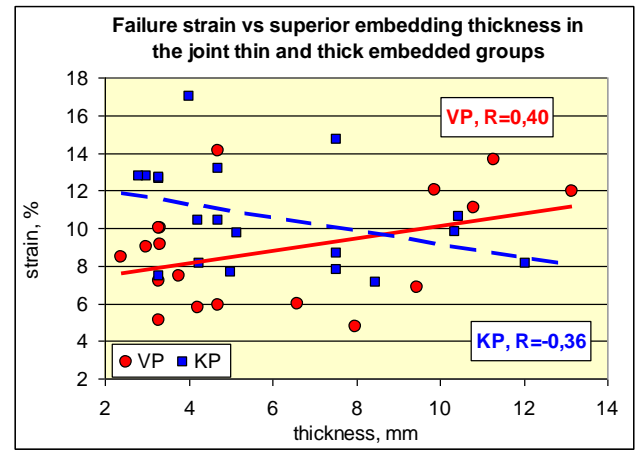

a)

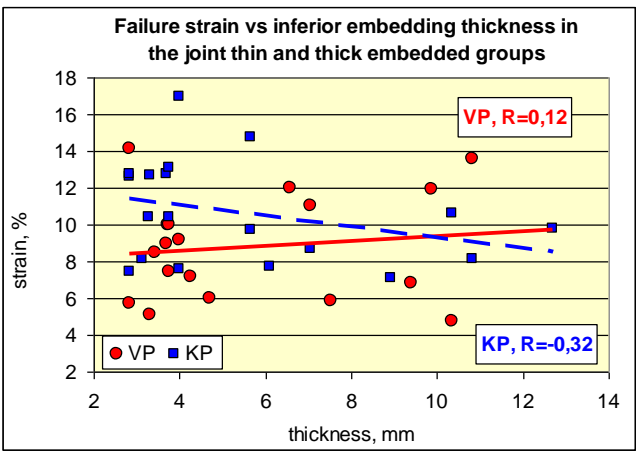

b)

Figure 4. Failure strain vs a) superior and b) inferior embedding thickness in VP and KP groups 


\begin{tabular}{|c|c|c|c|c|c|c|}
\hline $\begin{array}{c}\text { Failure strain vs } \\
\text { emb. thickness }\end{array}$ & VP1 & KP1 & VP2 & KP2 & VP & KP \\
\hline superior & 0.80 & -0.15 & 0.32 & -0.29 & 0.40 & -0.36 \\
\hline inferior & 0.20 & -0.25 & -0.14 & 0.25 & 0.12 & -0.32 \\
\hline mean & 0.63 & -0.21 & -0.09 & 0.25 & 0.29 & -0.34 \\
\hline
\end{tabular}

Table 5. Correlation between failure strain and superior, inferior and mean embedding thickness

Correlation between the failure compressive strains and the embedding thicknesses of thick and thin embedded VP and KP vertebrae can be seen in Figure 4 and Table 5. Surprisingly, the stiffness of VP vertebrae showed a considerable positive correlation with the embedding thickness in thick embedded groups, while there was no correlation in the thin embedded group, and in the joint thick and thin embedded groups a small negative correlation was shown. However, while the compressive strain of KP vertebrae showed no correlation in separated thin and thick embedded groups, for the joint groups a modest negative correlation was observed equally for the superior $(\mathrm{R}=-0.36)$, inferior $(\mathrm{R}=-0.32)$ and mean $(\mathrm{R}=-0.34)$ embedding thicknesses.

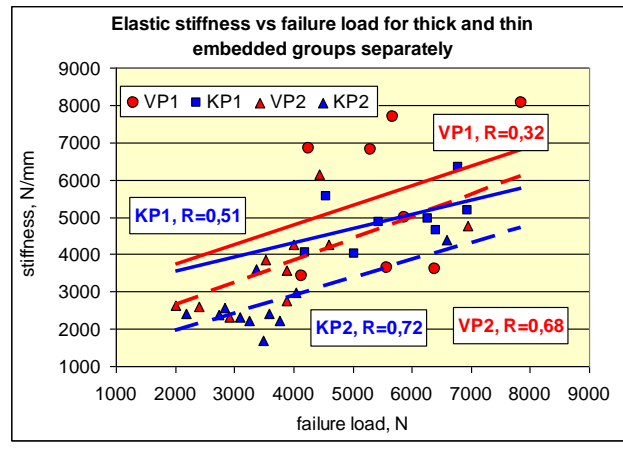

a)

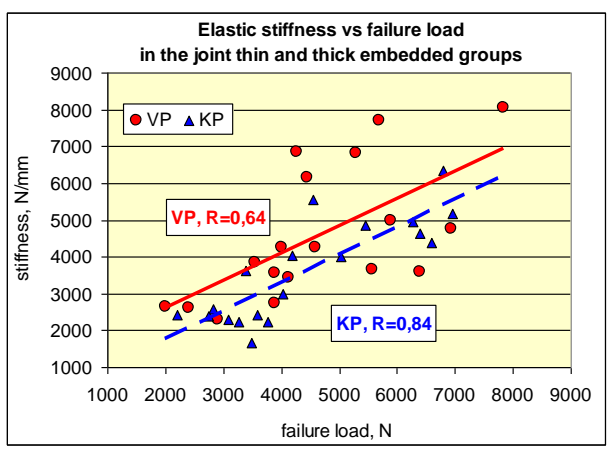

b)

Figure 5. Elastic stiffness vs failure load of VP and KP vertebrae a) in thick and thin embedded groups separately and b) in the joint thick and thin embedded groups

Correlation between the elastic stiffness and failure load of thick and thin embedded VP and KP vertebrae can be seen in Figures $5 a$ and $5 b$, for the separated and unified tick and thin embedded groups, respectively. In the thin embedded group both VP2 $(\mathrm{R}=0.68)$ and KP2 $(\mathrm{R}=0.72$, vertebrae had higher correlation between stiffness and failure load than in the thick embedded VP1 $(\mathrm{R}=0.32)$ and KP1 $(\mathrm{R}=0.51)$ groups. KP vertebrae had higher correlation in both the thick and thin or in the joined thick and thin embedded groups $(R=0.84)$ than VP vertebrae $(R=0.64)$. 


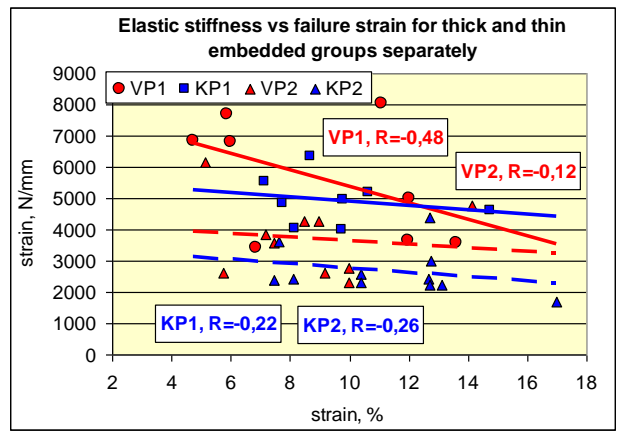

a)

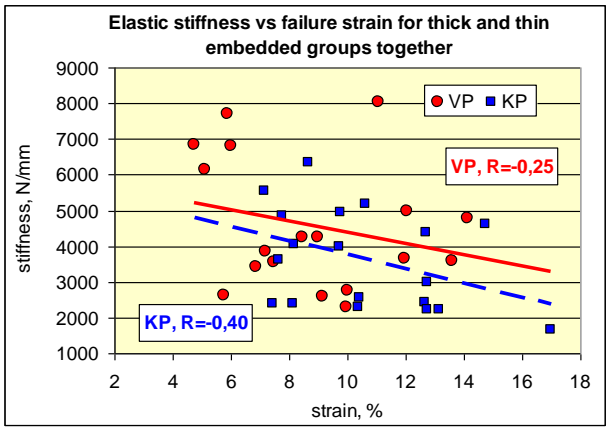

b)

Figure 6. Elastic stiffness vs failure strain of VP and KP vertebrae a) in thick and thin embedded groups separately and b) in the joint thick and thin embedded groups

Correlation between the elastic stiffness and failure strain of thick and thin embedded VP and KP vertebrae can be seen in Figures $6 a$ and $6 b$, for the separated and unified tick and thin embedded groups, respectively. Evidently, all correlations are negative. VP1 vertebrae in the thick embedded group showed a modest negative correlation, all other vertebrae showed a small negative correlation, In the unified thick and thin embedded groups both VP $(\mathrm{R}=-0,25)$ and KP $(\mathrm{R}=-0,40)$ vertebrae had a modest negative correlation between the compressive strain and the embedding thickness.

\subsection{The influence of the embedding thickness on the plastic damage process of a vertebral interbody device}

The results of the compressive mechanical test applied for the segments of interbody fusion, the failure loads, failure displacements and elastic stiffnesses are detailed $i^{4}$, and the related specimen-specific finite element method is presented in ${ }^{5}$. Here the analysis of the effect of embedding thickness by using case-specific finite element method was introduced for a single case of PMMA cement interbody device (PMMA-5 specimen in Table $1 a$ and $1 b$ in $^{4}$ ). The QCTbased finite element model of the specimen is seen in Figure 7.

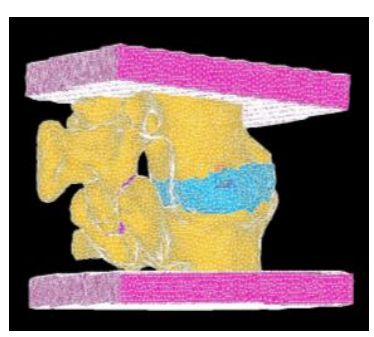

a)

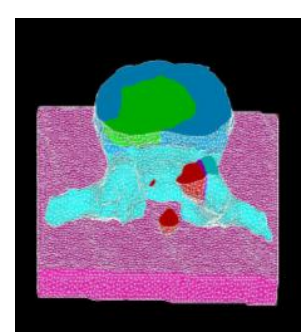

b)

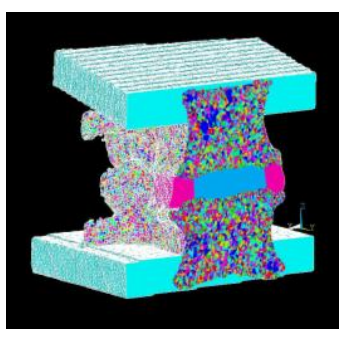

c)

Figure. 7. The QCT-based finite element model of the specimen of interbody fixation with PMMA cement interbody device (a); horizontal section through the disc with annulus remnant and cement spacer (b); and frontal section with the QCT- and BMD-based inhomogeneous cancellous and cortical bone (c) 
In the numerical experiment, first the lower plane of the inferior embedding layer was fully constrained, and $5 \mathrm{~mm}$ compressive displacement load was applied in 10 time steps on the upper surface of the superior embedding along the direction of the plane normal. The steps of the gradual elastic-plastic stain evolution procedure of the specimen are shown in Figure 8 for the original $10 \mathrm{~mm}$ thick superior and inferior embedding. As it was mentioned in the Method section for the embedding plastic material the elastic modulus was measured experimentally on small cylindrical samples and the obtained elastic modulus was used in the finite element analysis (Table 2). To avoid the effect of the embedding material, the loading procedure was performed by using a three times increased value for the embedding plastic, but the obtained load bearing and strain results were practically the same as seen in Figure 8.
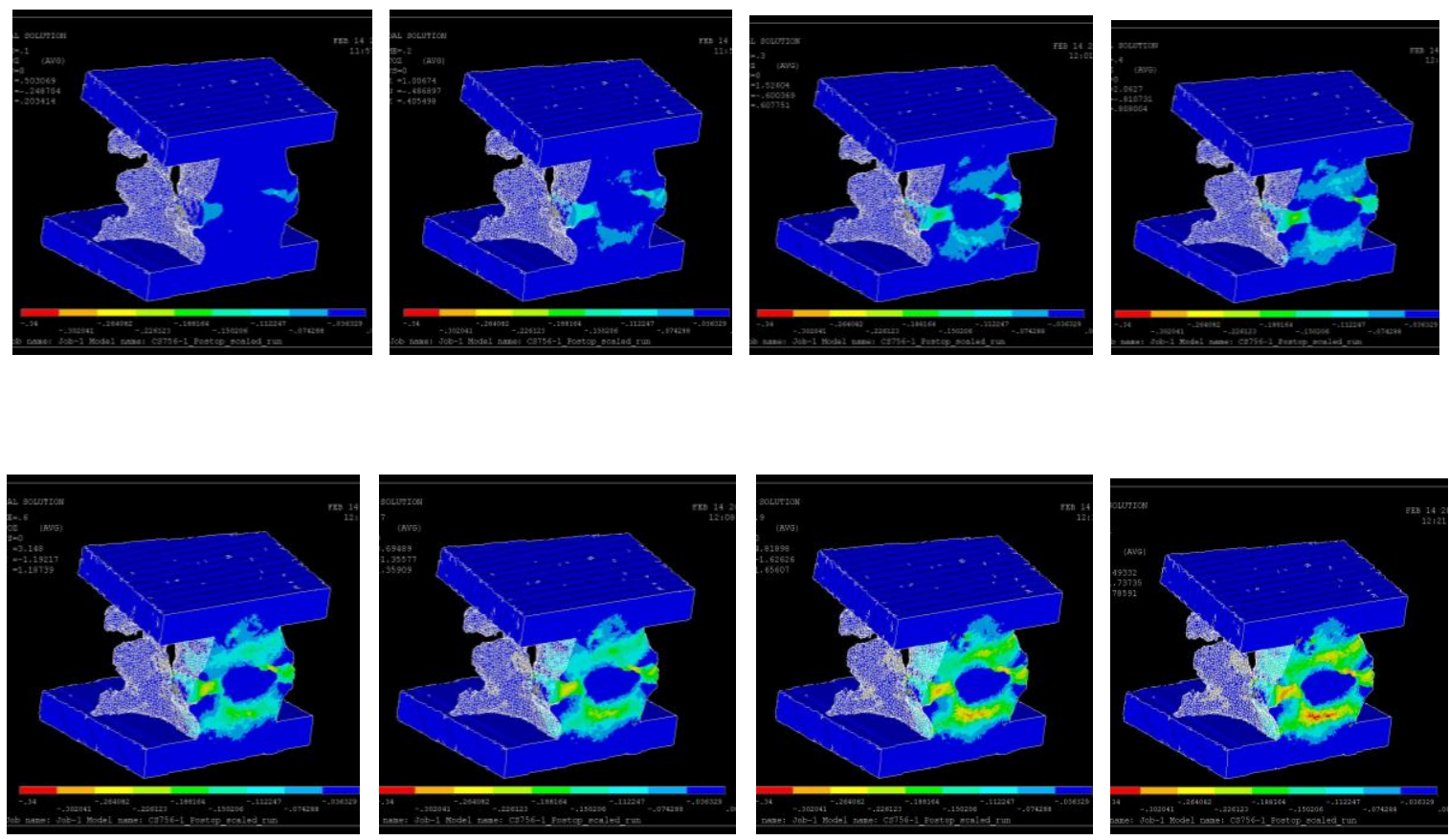

Figure 8. Elastic-plastic damage procedure with the developing failure zones

Since the damage is represented by the plastification, in Figure 9 the separated elastic and plastic strain zones are illustrated for the last step of loading procedure. Obviously, the maximal elastic strains occurred in the remnants of disc annulus while the maximal plastic strains developed in the trabecular bone, under the quasi rigid, non-deformable cement spacer. Thus, in the case of full $10 \mathrm{~mm}$ thick embedding, the damage zone started to develop just under the cement spacer, in the middle of the inferior vertebra, and the final failure zone situated at the same place.

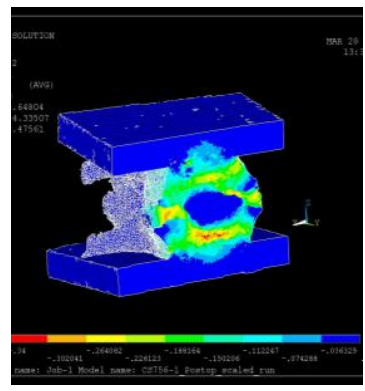

a)

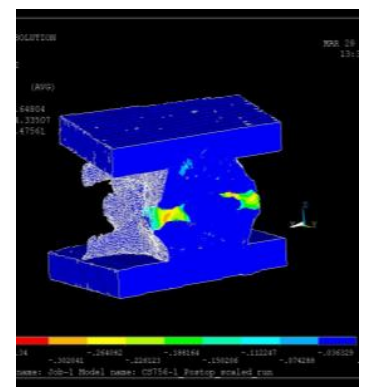

b)

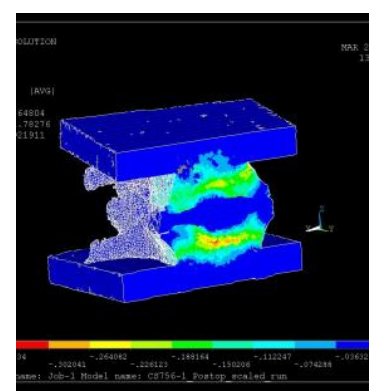

c)

Figure 9. Total (a) and separated elastic (b) and plastic (c) strain zones in the final state of loading 
For the slicing numerical experiment, the final location of the elastic-plastic strain zones of the last loading step is shown in Figure 10. Here the final elastic-plastic zones for the total thickness of $10 \mathrm{~mm}$, then for 7.5, 5.0, $2.5 \mathrm{~mm}$ and finally for the total removing of the embedding is illustrated. The last picture in Figure 10 shows the case of the inverse directional loading, with support on the top and displacement load on the bottom, acting upwards.

Since the damage can be represented by the evolution of plastic compressive strains during the loading, in Figure 11 the relating separated plastic strains, that is, the damage zones are distinguished. By thinning gradually the embedding, the failure zone that started to develop in the middle of the inferior vertebra, just under the cement spacer, moved upwards, with the final position closed to the top of the superior vertebra.

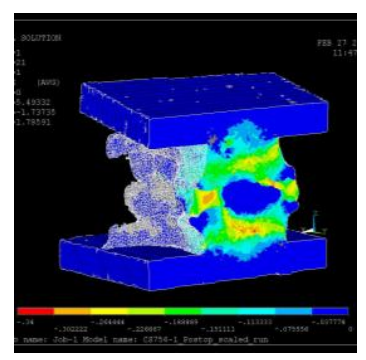

a)

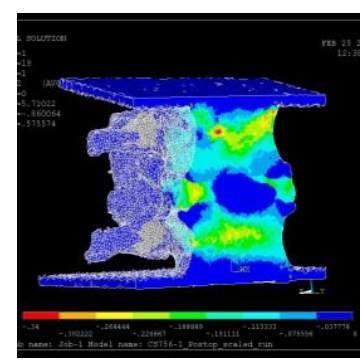

d)

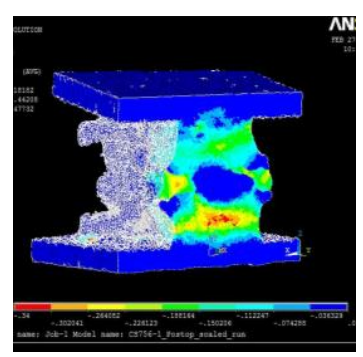

b)

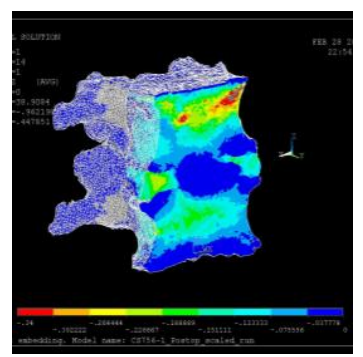

e)

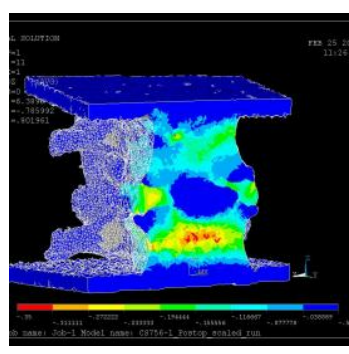

c)

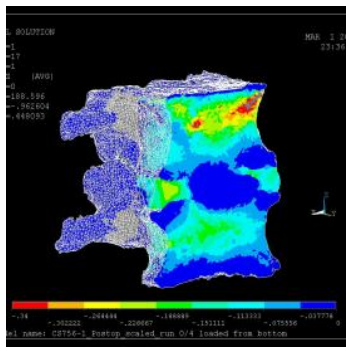

f)

Figure 10. Final damage zones in the case of a) $10 \mathrm{~mm}$, b) $7.5 \mathrm{~mm}$, c) $5.0 \mathrm{~mm}$, d) $2.5 \mathrm{~mm}$ and e) total remove of embedding, f) loaded upside down the case of total removed embedding

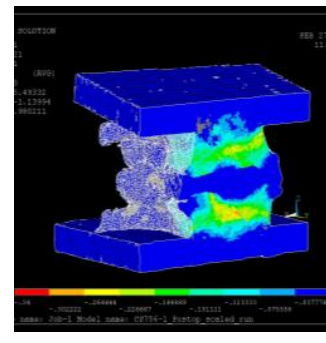

a)

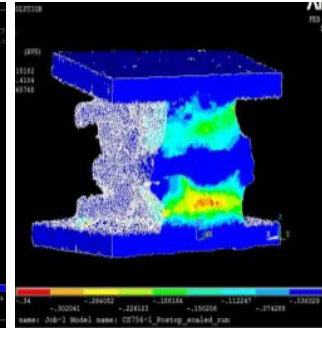

b)

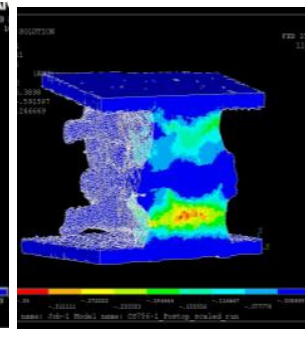

c)

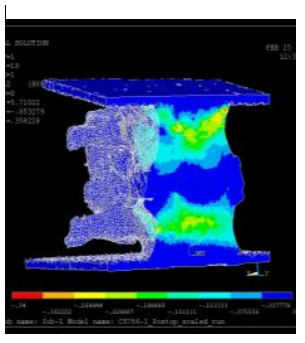

d)

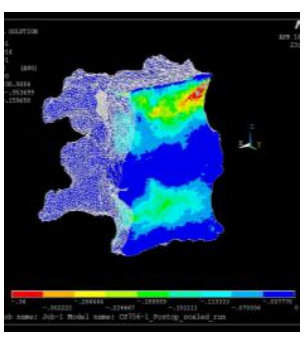

e)

Figure 11. The separated plastic strain domains of the final damage zones in the case of a) $10 \mathrm{~mm}, \mathrm{~b}) 7.5$ $\mathrm{mm}$, c) $5.0 \mathrm{~mm}$, d) $2.5 \mathrm{~mm}$ and e) total remove of embedding 


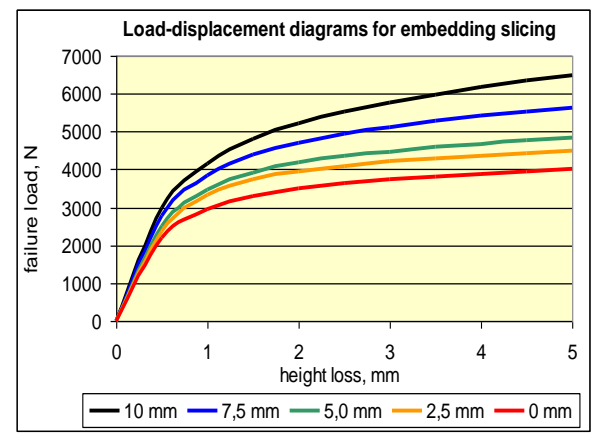

a)

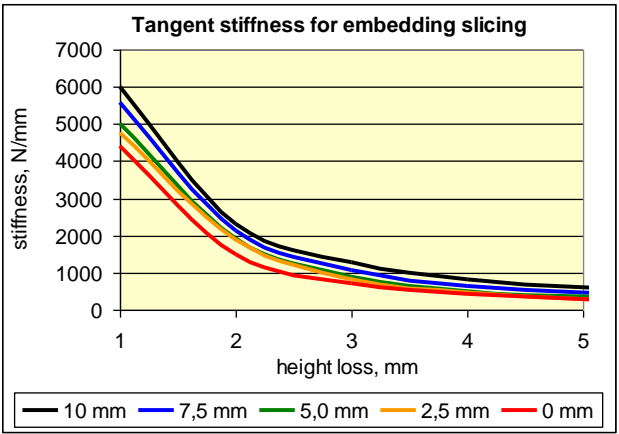

b)

Figure 12. Load-displacement diagrams (a) and tangent stiffness (b) for different embedding thicknesses

In Figure 12 the load-displacement diagrams and the gradually decreasing tangent stiffnesses are shown for the slicing steps of embedding. By the total remove of embedding, the load bearing of the segment at the last loading step gradually decreased by about $40 \%$, by $18,14,8$ and $11 \%$ in each slice reduction step, subsequently, related to the previous value. The related stiffness decreased by $65 \%$ in $28,33,22$ and $9 \%$ steps.

\section{Discussion and Conclusion}

Mechanical results of vertebral augmentation highly depended on the embedding thickness. We can state that the thin embedding makes the specimens more sensitive to the differences between the mechanical results of VP and KP vertebrae than the thick one, as shown in Figure 1, namely, in thin embedding the differences in failure load, displacement and stiffness between VP and $\mathrm{KP}$ groups are larger. Compared to the native vertebrae without embedding in3, the failure load was about $80 \%$ higher in the thick and only $10-20 \%$ higher in thin embedded group. The embedding thickness significantly affected most of the mechanical results inside the augmentation type as well. The failure load and stiffness in the thin embedded groups was significantly smaller both VP and KP vertebrae compared to the thick embedded groups (Figures $1 a, 1 b)$.

The failure load is proportional with the embedding thicknesses equally for VP and KP vertebrae (Figure 2). However, this fact can be proved only in the joined thin and thick embedded groups. The reason is that the measured results are sensitive only to the larger thickness differences. However, the elastic stiffness only in the case of KP vertebrae is proportional with the embedding thicknesses, while the stiffness of VP vertebrae shows a great uncertainty in relation with the embedding thickness (Figure 3). The reason may be that due to the kyphoplasty a regular structure is formed that is partly supported by the embedding, while the stiffness of vertebroplasty is based mainly on the bone quality and structure. Indeed, as seen in, ${ }^{3}$ there was higher bone quality in the thick embedded group than in the thin embedded one, so the correlation of the stiffness of VP vertebrae with bone quality was negative in thick and strongly positive in thin embedded group where the cement augmentation could fill in the osteoporotic 
gaps. Similar regularity is found in the inverse proportionality between the failure compressive strains and the embedding thicknesses for KP vertebrae, and uncertainty for VP vertebrae (Figure 4). The reason may be the same as before.

Failure load and elastic stiffness are evidently proportional with each other. It is realized in both embedded groups, for both types of augmentation, however, better for the thin embedded group and better for KP vertebrae (Figure 5). The also evident inverse relationship between stiffness and deformability manifests itself better for KP augmentation again (Figure 6). The reason may be the clear and definite structure of kyphoplasty again, in comparison with the bone quality-dependent, irregularly distributed vertebroplasty. At the same time, in a comprehensive analysis the structural differences can better be distinguished in a thin embedded environment.

We can conclude that since the thin embedding is closer to the anatomic situation, the values of thin embedded group can be accepted as numerical results, however, the tendencies can be confirmed by the results of thick embedded group.

The specimen-specific finite element simulation of the failure process of the interbody fixation demonstrated a great influence of the embedding thickness on the mechanical characteristics. By means of the slicing method, beside the gradual decrease of the failure load and stiffness of specimens by slicing the embedding even thinner and thinner, the migration of the damage zones within the vertebral cancellous bone of the fixed segment could be presented. By the initial total embedding thickness the damage zone starts and develops under the cement spacer in the middle trabecular bone domain of the inferior vertebra. As the embedding become thinner and thinner, the position of the damage zone moves upwards, finally to the top of the upper vertebra (Figure 11). At the same time, the load bearing and stiffness of the segment gradually decreases (Figure 12). By applying the support and load in the inverse direction, the damage process and the numerical results are the same. The approximately $40 \%$ difference in the compressive strength between the $10 \mathrm{~mm}$ thick and no embedding is in agreement with the conclusion of 8 that the systematic underestimation error in the platens compression test can be in the range of $20-40 \%$, however, for trabecular bone specimens, for end-artifact errors.

The damage inside the bone is manifested by plastic strains. In the case of no embedding with top loading, the damage starts in the upper region of the superior vertebra, closed to the loading region, according to the Saint-Venant principle. The reason may be the missing horizontal support at the ends of the specimens that - in the case of embedding - hinders the development of plastic strains. Theoretical analysis ${ }^{9}$ of the experimental artifacts in the compressive tests of trabecular bone concludes that without friction at the interface between the test machine and the specimen always underestimates Young's modulus, thereby reducing the accuracy of the test. Similarly, 1 found that the assumption of a frictionless boundary condition in the parallel plate compression loading configuration was a significant source of error that could be overcome with the use of rigid end caps.

We can conclude that by evaluating the results of any mechanical tests, the thickness of the embedding must be considered, and when is possible, the results must be checked by casespecific finite element analysis. 


\section{REFERENCES}

1. Jacobs CR, Davis BR, Rieger CJ, Francis JJ, Saad M, Fyhrie DP. The impact of boundary conditions and mesh size on the accuracy of cancellous bone tissue modulus determination using large-scale finiteelement modeling, Journal of Biomechanics, 1999;32(11):1159-64.

2. Bevill G, Eswaran SK, Farahmand F, Keaveny TM. The influence of boundary conditions and loading mode on high-resolution finite element-computed trabecular tissue properties, Bone, 2009;44:573-8.

3. Kurutz M, Jakab G, Varga P, Varga PP. Biomechanical evaluation of vertebroplasty and kyphoplasty by uniaxial compressive test, Biomechanica Hungarica, 2013;VI(1):311-22.

4. Kurutz M, Csákány T, Varga P, Varga PP. Biomechanical evaluation of interbody devices by uniaxial compressive test: PEEK spacers versus PMMA cement spacers, Biomechanica Hungarica, 2013;VI(1):259-71.

5. Varga P, Nédli P, Csákány T, Kurutz M, Varga PP. Biomechanical evaluation of two different vertebral interbody devices by using QCT-based case-specific nonlinear finite element models: A preliminary report, Biomechanica Hungarica, 2013;VI(1):175-84.

6. Kopperdahl DL, Morgan EF, Keaveny TM. Quantitative computed tomography estimates of the mechanical properties of human vertebral trabecular bone, J Orthop Res, 2002;20:801-5.

7. Mirzaei M, Zeinali A, Razmjoo A, Nazemi M. On prediction of the strength levels and failure patterns of human vertebrae using quantitative computed tomography (QCT)-based finite element method. J Biomech, 2009;42(11):1584-91.

8. Keaveny TM, Pinilla TP, Crawford RP, Kopperdahl DL, Lou A. Systematic and random errors in compression testing of trabecular bone, J Orthop Res, 1997;15:101-10.

9. Keaveny TM, Borchers RE, Gibson LJ, Hayes WC. Theoretical analysis of the experimental artifact in trabecular bone compressive modulus. J Biomech, 1993;26:599-607.

The authors gratefully acknowledge the Hungarian Scientific Research Fund OTKA for providing financial support in the frame of the grant $K$-075018. The authors are grateful to Lajos Borbás and Gábor Szebényi for their help in laboratory experiments. 